\title{
Growth of Single-Walled Carbon Nanotubes by Plasma CVD
}

\author{
Toshiaki Kato and Rikizo Hatakeyama \\ Department of Electronic Engineering, Tohoku University, Sendai 980-8579, Japan \\ Correspondence should be addressed to Toshiaki Kato, kato12@ecei.tohoku.ac.jp
}

Received 10 September 2010; Accepted 1 December 2010

Academic Editor: A. M. Rao

Copyright ( $) 2010$ T. Kato and R. Hatakeyama. This is an open access article distributed under the Creative Commons Attribution License, which permits unrestricted use, distribution, and reproduction in any medium, provided the original work is properly cited.

\begin{abstract}
Recent research in plasma chemical vapor deposition (CVD) for single-walled carbon nanotube (SWNT) growth has achieved low-temperature synthesis, individually freestanding formation, and structure control of diameter, chirality, and length. Detailed growth kinetics of SWNTs are revealed using a combination of techniques for plasma control and nanomaterial analysis. Plasma CVD also allows tube metallicity to be controlled by tuning the mean diameter of SWNTs. This plasma CVD progress contributes to the next stage of nanotube fabrication, which is required for practical use of SWNTs in a variety of applications.
\end{abstract}

\section{Introduction}

One-dimensional single-walled carbon nanotubes (SWNTs) are potential materials for future nanoelectronics [1-5]. Since the electronic and optical properties of SWNTs strongly depend on their structure, such as diameter, chirality, and length, the selective synthesis of SWNTs with desired structures is a major challenge in nanotube science and applications. SWNT growth was first achieved by arc discharge in 1993 [1]. Several growth techniques have been developed since then, including laser ablation [6] and chemical vapor deposition (CVD) [7]. Since it is possible to grow SWNTs at a specific position on a substrate by patterning a catalyst, CVD has attracted much attention in nanoelectronics applications. In general, CVD can be divided into two types: thermal CVD [7-9] and plasma CVD [10-13]. Due to the strong electric fields in plasma sheaths, nanotubes grown by plasma CVD tend to have an individually and vertically freestanding shape [11,14-16]. Thermal CVD decomposes carbon source gases using thermal energy. In contrast, in plasma CVD, the source gas decomposition is effectively carried out by electron impact with no additional thermal energy; hence, the growth temperature is significantly lower compared to that of thermal CVD [17-19]. Despite these benefits of plasma CVD, it is difficult to control the structure of SWNTs by plasma CVD because there are many unknown factors in plasma, such as ion density, ion energy, radical species, radical densities, and sheath electric field, which restrict the potential application of plasma CVD in nanotube science. Based on previous studies, SWNT growth by plasma CVD has been significantly improved in recent years. Here, we give a brief overview of recent achievements in SWNT growth by plasma CVD.

\section{Freestanding Single-Walled Carbon Nanotube Growth}

The potential of plasma CVD for nanotube growth was first demonstrated by Ren et al. in 1998 [11]. Vertically and individually aligned multiwalled carbon nanotubes (MWNTs) are grown by plasma CVD. Since carbon nanotubes (CNTs) grown by thermal CVD are known to form a spaghettilike entangled shape, the well-aligned growth of CNTs by plasma CVD makes it an attractive CNT-growth method that may solve the integration issue in CNT-based nanoelectronics. However, plasma CVD is limited to the production of MWNTs; SWNTs, which have superior electrical and optical characteristics compared with MWNTs, have not been successfully produced by plasma CVD. The growth of SWNTs by plasma CVD was first reported by our group in 2003 [20]. SWNTs are grown by plasma CVD using a zeolite as a catalyst support. Zeolites are nanoporous materials known to maintain small catalyst particle sizes on their rough surfaces, even under high-temperature conditions. 


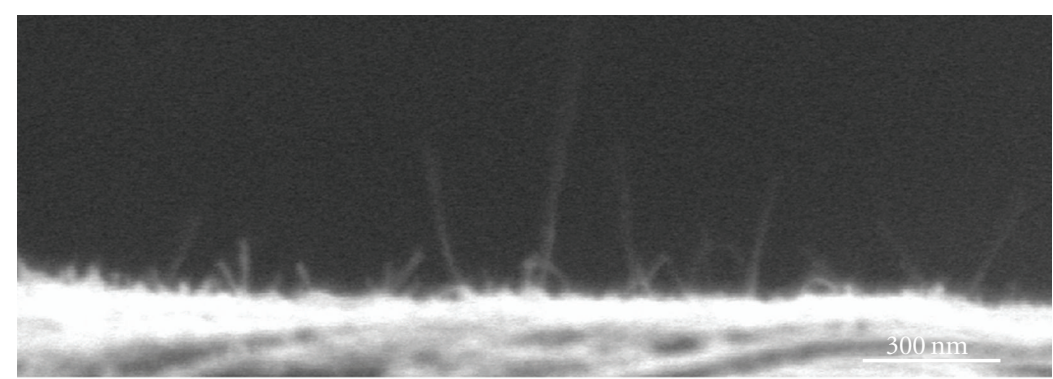

(a)

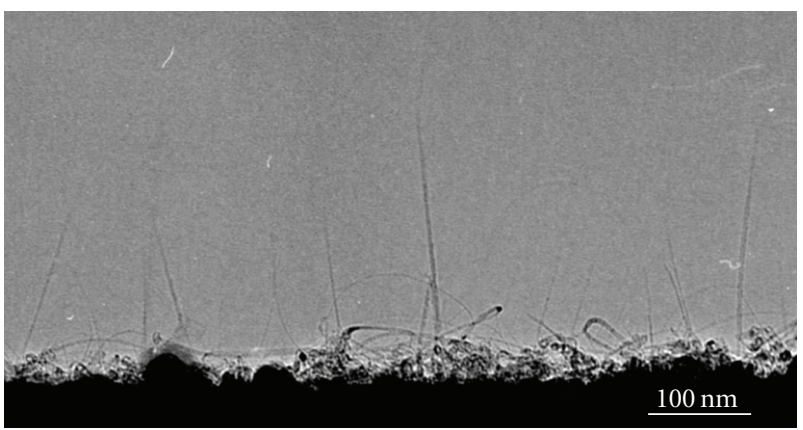

(b)

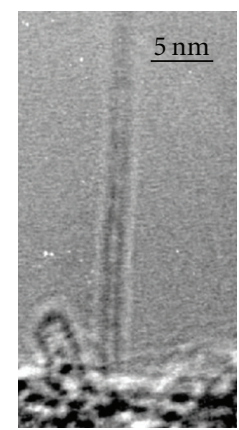

(c)



(d)

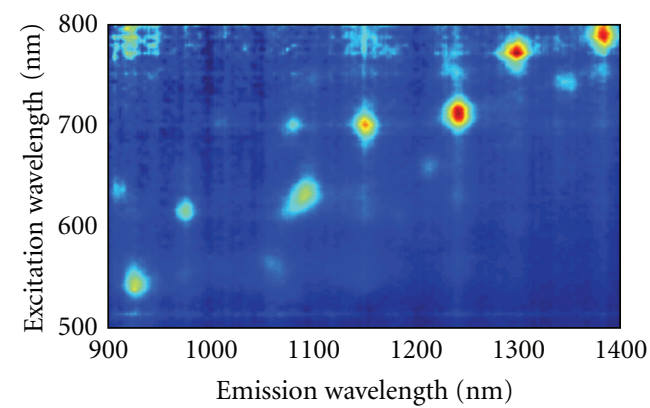

(e)

FIGURE 1: (a) SEM and (b, c) TEM images of freestanding individual SWNTs. (d) Raman scattering spectrum of freestanding individual SWNTs. The inset of (d) shows the RBM region. (e) PLE map from as-grown freestanding SWNTs with no dispersion process.

Thus, certain plasma effects might enhance catalyst particle aggregation during plasma CVD, which could be the main reason why SWNTs could not be grown by plasma CVD. It is thought that catalyst particle aggregation is enhanced due to high-energy ions attacking the catalyst. In general, ions in plasma are accelerated through the potential drop between space potentials in the plasma and substrate biases. The minimum value of this potential drop is determined by the electron temperature in the plasma. Thus, low electrontemperature plasma can significantly decrease the energy of ions arriving at the substrate. Since the diffusion region in plasma is known to have very low electron temperatures, we used the diffusion plasma to decrease the energy of ions attacking the catalyst to below a few eV. SWNT growth under the diffusion plasma region occurs on a flat substrate without using catalyst support materials [21-23]. Thus, the critical element promoting catalyst aggregation is high-energy ion bombardment. Interestingly, SWNTs grown by diffusionplasma CVD have the well-aligned freestanding form, that is, all SWNTs are individually and vertically standing on the flat substrate. Figures 1(a)-1(d) show a typical scanning electron microscope (SEM) image (Figure 1(a)), low-magnification (Figure 1(b)) and high-magnification (Figure 1(c)) transmission electron microscope (TEM) images, and Raman scattering spectra (Figure 1(d)) of freestanding SWNTs. Relatively high-quality SWNTs were grown with the individually freestanding form, and this alignment can be obtained by the plasma-sheath electric field. Based on our estimation, the rotation energy of the dipole moment in SWNTs is much higher than the thermal energy, which disturbs the tube alignment [23]. This indicates that individual SWNTs can be aligned along the electric field. Owing to their unique asgrown state, it is possible to directly detect photoluminescence (PL) spectra from the as-grown freestanding SWNTs on the substrate (Figure 1(e)) [24]. This is a remarkable advantage for optoelectrical applications and fundamental studies toward chirality control, which will be discussed later.

\section{Low-Temperature Growth}

In addition to the alignment control of CNTs, lowtemperature growth is another benefit of plasma CVD [1719]. Since the carbon source gasses are decomposed by the electron impact in plasma, the growth temperature can be lower than that of conventional thermal CVD. Although the quality of the graphite network in a tube structure is not high and a carbon nanofiber-like structure can be formed, the growth temperature of MWNTs is below $120^{\circ} \mathrm{C}$ with plasma CVD [17]. For SWNTs, the lowest reported growth temperature is $\sim 400^{\circ} \mathrm{C}[18]$. Low-temperature growth enabled us to directly grow CNTs on low-melting point materials, such as plastics and polymers. Hence, the application field of CNTs is broadened by improving the low-temperature growth of CNTs using plasma CVD.

\section{Growth Mechanism}

To achieve structure-controlled growth of SWNTs by plasma CVD, it is important to understand the growth kinetics of 


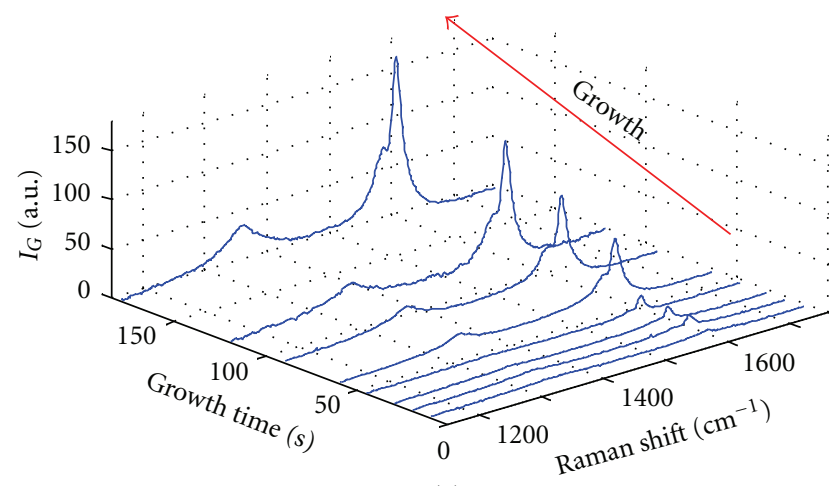

(a)

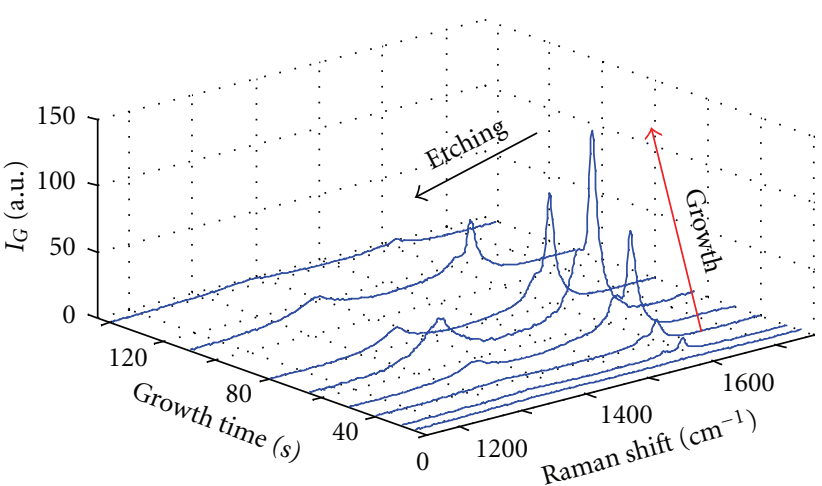

(b)

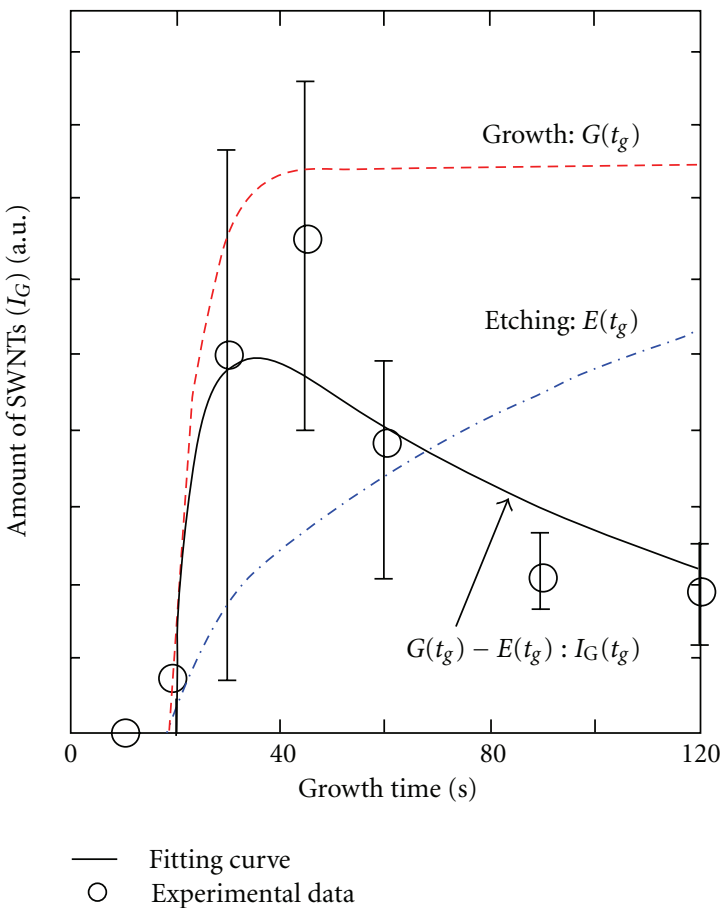

(c)

FIgURE 2: Raman spectra of SWNTs as a function of growth time. (a) $P_{\mathrm{RF}}=40 \mathrm{~W}$ and (b) $P_{\mathrm{RF}}=100 \mathrm{~W}$. (c) Comparison between the experimental data and the fitting curve of (1).

SWNTs during plasma CVD and elucidate the effects of plasma in detail.

For thermal CVD, it is reported that the growth kinetics of SWNTs can be expressed by $I_{\mathrm{G}}=I_{0}\left[1-\exp \left\{-\left(t_{g}-\right.\right.\right.$ $\left.\Delta t) / \tau_{\text {gro }}\right\}$ ] (normal equation) [25], where $I_{G}, I_{0}, t_{g}, \Delta t$, and $\tau_{\text {gro }}$ denote the $G$-band intensity in Raman scattering spectra ( $\sim$ amount of SWNTs), saturated $I_{G}$, growth time, incubation time, and relaxation time of the growth, respectively. Figures 2(a) and 2(b) show typical Raman scattering spectra of SWNTs as a function of growth time under different radio frequency (RF; $13.56 \mathrm{MHz})$ power $\left(P_{\mathrm{RF}}\right)$ conditions. The experimental results of the damage-free growth (Figure 2(a)) match well with this equation, indicating that our estimation of the amount of SWNTs with $I_{G}$ is reliable. However, there is obviously no formula that can describe growth kinetics by including an etching effect, as shown in Figure 2(b). To express this phenomenon, we assumed that the growth mode could be described with a balance equation, $I_{\mathrm{G}}=$ $G\left(t_{g}\right)-E\left(t_{g}\right)$, where $G\left(t_{g}\right)$ and $E\left(t_{g}\right)$ are growth and etching functions, respectively. When the etching effect is weak and negligible, the growth kinetics can be described with the aforementioned normal equation, indicating that $G\left(t_{g}\right)$ gives results that are similar to the normal equation. One of the most important outcomes of this study is the description of $E\left(t_{g}\right)$. Since carbon atoms are etched only when atoms or molecules attach themselves to the carbon atoms in SWNTs, the probability of the etching reaction can be simplified in terms of an adsorption reaction. Langmuir's adsorption isotherm, a well-known equation, can be expressed as $d \theta / d t=\alpha P(1-\theta)$, where $\theta, t, \alpha$, and $P$ denote the percentage of covered area, reaction time, adsorption efficiency, and pressure of the adsorbate, respectively. The Langmuir's equation has been used in a wide range of fundamental scientific studies to understand chemical adsorption reactions [26]. In our study, $\theta$ corresponds to the etched area against the graphite sheet of SWNTs, that is, $\theta=E\left(t_{g}\right) / G\left(t_{g}\right)$. Since the solution of the Langmuir's equation is $\theta\left(t_{g}\right)=$ $1-\exp \left(-t_{g} / \tau_{\text {etc }}\right)$, where $\tau_{\text {etc }}=1 / \alpha P$ is the relaxation time of the etching reaction, $E\left(t_{g}\right)$ results in the following equation: $E\left(t_{g}\right)=G\left(t_{g}\right)\left\{1-\exp \left(-t_{g} / \tau_{\text {etc }}\right)\right\}$. According to the aforementioned equations, an advanced growth equation can be established as

$$
I_{\mathrm{G}}=I_{0}\left[1-\exp \left\{\frac{-\left(t_{g}-\Delta t\right)}{\tau_{\text {gro }}}\right\}\right]\left\{\exp \left(\frac{-t_{g}}{\tau_{\text {etc }}}\right)\right\} .
$$

Figure 2(c) compares the experimental result of Figure 2(b) with the fitting curve from (1). The fitting curve agreed well with the experimental result.

Since there are several important fitting parameters related to growth kinetics of SWNTs in (1), such as $\Delta t$, 
etching efficiency, and catalyst lifetime, it is possible to precisely understand the correlation between plasma parameters and growth parameters, leading to improved structurecontrolled growth of SWNTs by plasma CVD [27]. Our systematic experiment, combined with this growth-equation study, reveals that unique reactive-ion etching occurred during the growth of SWNTs under specific plasma conditions in plasma CVD [27].

\section{Structure-Controlled Growth of SWNTs}

The structure of SWNTs, including diameter, chirality, and length, strongly influences their electrical and optical properties; therefore, it is important to precisely control the structure of SWNTs. Here, we discuss recent progress in the structure-controlled growth of SWNTs by plasma CVD.

5.1. Diameter Control. The band gap is known to be inversely proportional to tube diameter; thus, controlling the tube diameter is very important for electrical and optical applications. Here, we present our experimental results for diameter tuning of SWNTs based on gas-phase control in plasma CVD [28].

Figures 3(a)-3(d) show photoluminescence-excitation (PLE) maps of as-grown SWNTs produced at different gas pressures. Note that all PLE measurements were carried out immediately after the growth process to prevent the freestanding SWNTs from forming bundles, which cause significant PL changes [24]. Peaks in the PLE map at high growth pressures (Figure 3(a)) appeared in the range of long excitation and emission wavelengths. The peak positions shifted to the region of short excitation and emission wavelengths with decreasing growth pressure (Figures 3(b)$3(d))$. Since each peak corresponds to a different chirality in the sample, and smaller-diameter SWNTs appeared in the shorter wavelength region, the peak-position shifts in the PLE map indicate that the diameter distribution of SWNTs is strongly influenced by growth pressure. Thus, lower pressure results in smaller SWNT diameters. This diameter dependence on the growth pressure is also reflected in Raman scattering spectra of SWNTs grown at different growth pressures. Figure 3(e) shows that peak positions of the radial breathing mode (RBM) clearly shifted from higher to lower wavenumbers with increasing growth pressure. The RBM peak position and the SWNT diameter are known to have a close correlation, $\omega=248 / d$ [29], where $\omega$ and $\mathrm{d}$ are the RBM peak position $\left(\mathrm{cm}^{-1}\right)$ and diameter $(\mathrm{nm})$, respectively. This result is fairly consistent with the PLE result shown in Figures 3(a)-3(d). The typical pressure range where SWNTs can be grown is from $30 \mathrm{~Pa}$ to $650 \mathrm{~Pa}$ and depends on the $P_{\mathrm{RF}}$ used for the plasma generation. Although the absolute intensity of the $G$-band in Raman scattering spectra decreased in the low- or high-pressure range, the $G$-band to $D$-band ratio was almost constant. This indicates that the quality of SWNTs should be independent of the pressure range, whereas the density of SWNTs depends on the pressure. When we increased the input $P_{\mathrm{RF}}$, it was possible to grow SWNTs, even below $30 \mathrm{~Pa}$, indicating that a lack of hydrocarbon supply is significant under low-pressure conditions. Hence, an additional input $P_{\mathrm{RF}}$ is required to increase the density of active species used for the growth of SWNTs. Since the pressure during the heating and growth was the same in our growth process, the process pressure affected both the heating and the growth process. Based on this systematic investigation, we believe that the catalyst particle size increased due to aggregation after high-pressure annealing, which resulted in the growth of large-diameter SWNTs. The density of reactive hydrocarbon radicals and ions should increase under higher growth pressure conditions. Under high carbon supply conditions, a small catalyst can be deactivated by an oversupply of hydrocarbons, causing the population of small-diameter SWNTs to decrease. Therefore, the heating pressure is an important parameter that controls the catalyst particle size distribution, which directly influences the diameter of SWNTs. The pressure during plasma CVD is also important for a narrow SWNT diameter distribution [28].

5.2. Chirality Distribution Control. The chirality of SWNTs directly determines their electronic and optical properties; thus, selective synthesis of SWNTs with desired chiralities is a major challenge in nanotube science and applications [3, $30,31]$. Some progress has been made with silica-supported CoMo [32] and zeolite-supported FeCo [33] catalysts. FeRu [34] and FeNi [35] catalysts have also been developed to achieve narrow chirality distributions. Interestingly, all syntheses resulting in narrow chirality distributions have involved magnetic catalysts. The main obstacle to research on intrinsic magnetic properties of SWNTs is residual ferromagnetic catalyst particles; thus, SWNT growth with nonmagnetic catalysts is beneficial. Despite recent improvements in SWNT growth with nonmagnetic catalysts [3639 ], diameter and chirality $(n, m)$ distribution control with nonmagnetic catalysts is still required for fundamental studies and a variety of applications [40].

In this subsection, we discuss a recent achievement in the growth of SWNTs with narrow chirality distributions using an Au catalyst and plasma CVD [41, 42]. PLE mapping [43] was used to assign $(n, m)$ of SWNTs grown from the Au catalyst at different $\mathrm{H}_{2}$ concentrations (Figures 4(a)-4(c)). The total pressure was kept at $50 \mathrm{~Pa}$ by adjusting the pumping rate of the rotary pump throughout this experiment. Lower $\mathrm{H}_{2}$ concentrations ( 0 and $3 \mathrm{sccm}$ ) led to larger diameters and wider $(n, m)$ distributions with $(6,5),(7,5),(7,6),(8,4)$, $(8,6)$, and $(8,7)$ (Figures $4(\mathrm{a})$ and $4(\mathrm{~b}))$. On the other hand, the $7-\mathrm{sccm} \mathrm{H}_{2}$ concentration yielded the narrowest $(n, m)$ distribution with a dominant peak corresponding to the $(6,5)$ tube (Figure 4(c)). The UV-Vis-NIR optical absorbance spectra of Au-plasma CVD SWNTs grown at the 7-sccm $\mathrm{H}_{2}$ flow rate showed one dominant peak in the first van Hove $E_{11}$ range $(900-1400 \mathrm{~nm})$ corresponding to SWNTs with $(6,5)$ chirality (Figure $4(\mathrm{~d})$ ). Since clear metallic SWNT peaks were not observed in the UV-Vis-NIR spectra (Figure 4(d)), the concentration of metallic SWNTs was lower than that of the generally grown SWNTs. The RBM in the Raman spectra measured with 632.8-nm and 778-nm lasers on the same 


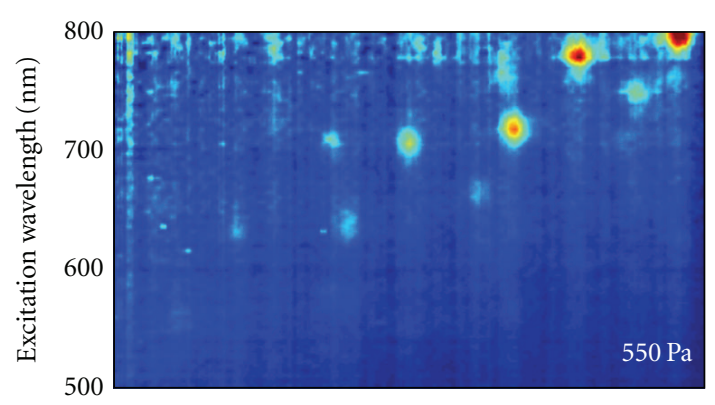

(a)

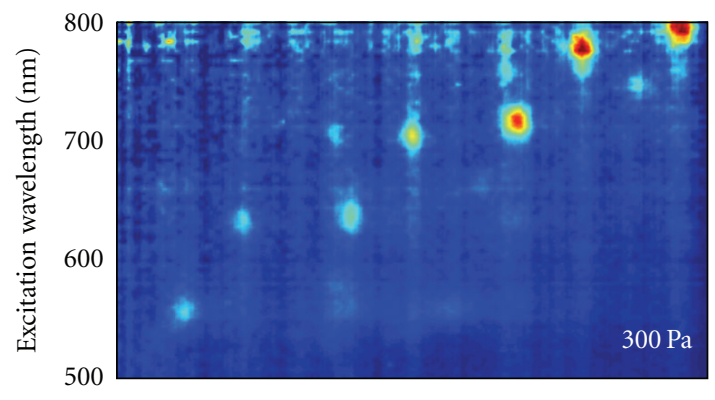

(b)

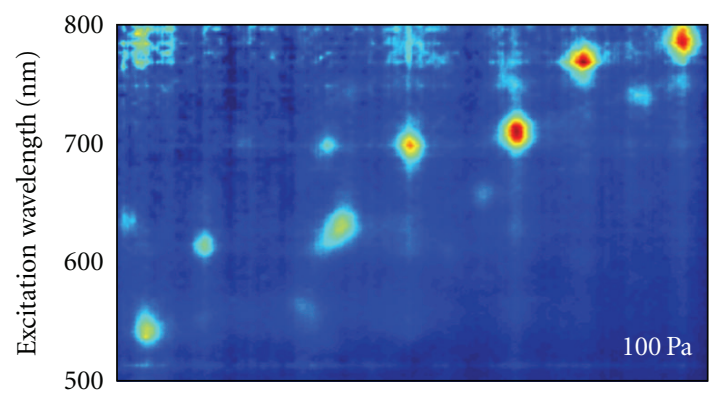

(c)

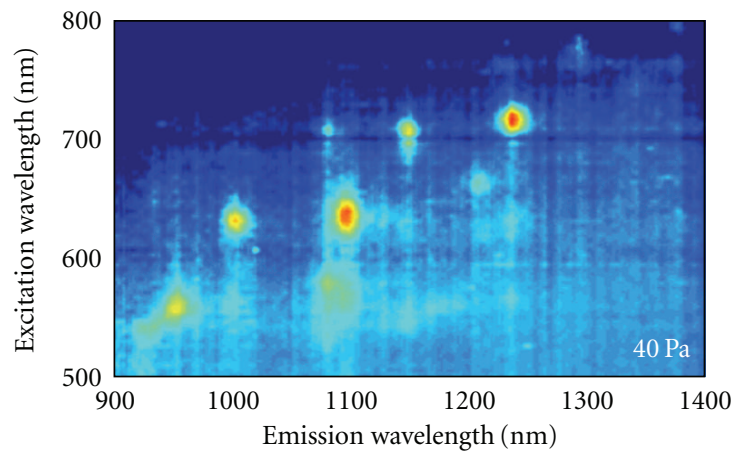

(d)

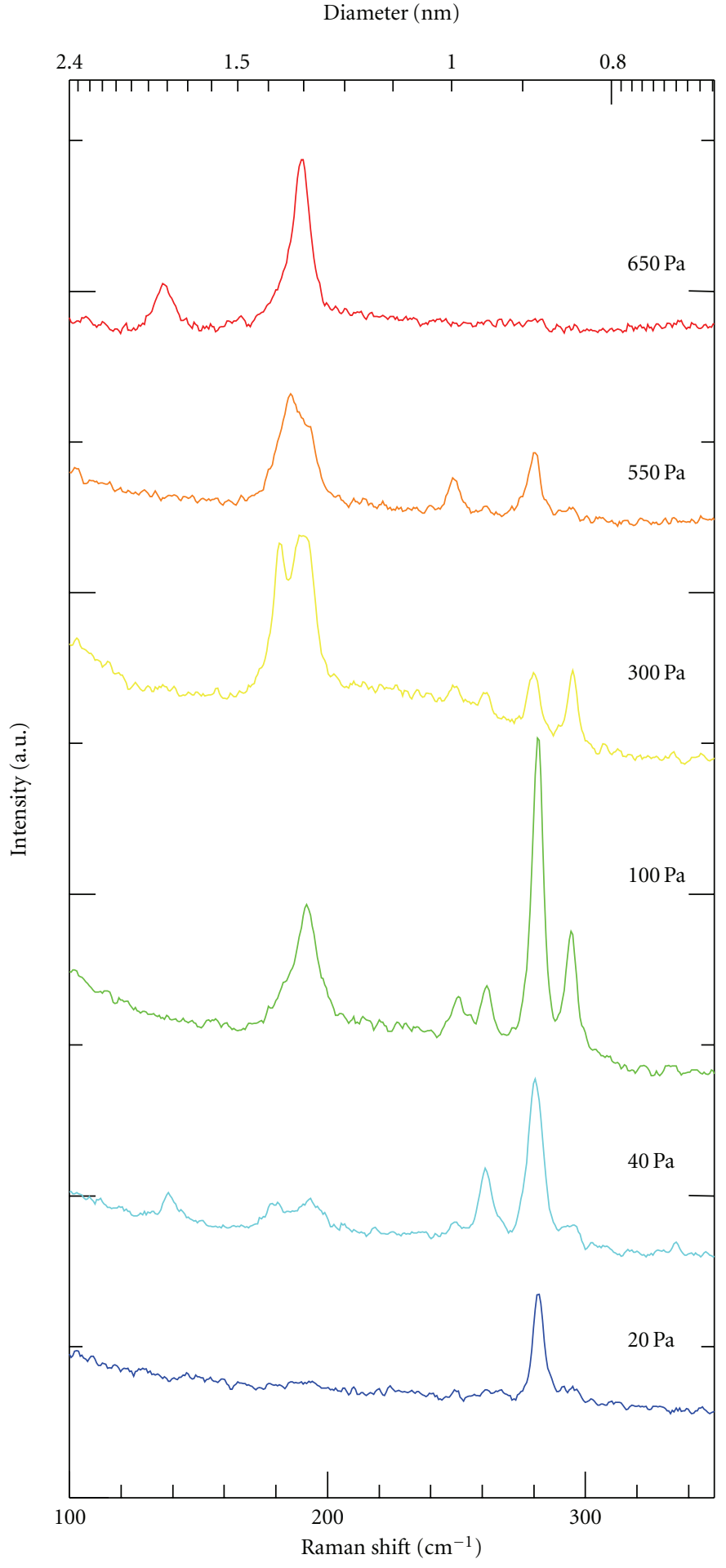

(e)

FIgURE 3: (a)-(d) PLE maps of as-grown freestanding SWNTs grown under different pressures ((a) $550 \mathrm{~Pa}$, (b) $300 \mathrm{~Pa}$, (c) $100 \mathrm{~Pa}$, and (d) $40 \mathrm{~Pa}$ ). (e) Dependence of the RBM in Raman scattering spectra on growth pressure for as-grown freestanding SWNTs.

SWNT sample also confirmed the growth of the $(6,5)$ SWNTs (Figure $4(\mathrm{e})$ ). This is the first result showing narrow chirality distributions for SWNTs grown from a nonmagnetic catalyst.

To elucidate the effects of $\mathrm{Au}$ and plasma CVD on the narrow chirality distribution, other combinations of catalysts and CVD methods were systematically investigated. Based on the PLE analysis, SWNTs grown by the Fe catalyst with plasma CVD (Fe-plasma CVD) did not show a clear correlation between the $\mathrm{H}_{2}$ flow rate and the chirality distribution, which was broader than that of SWNTs grown by Au-plasma 


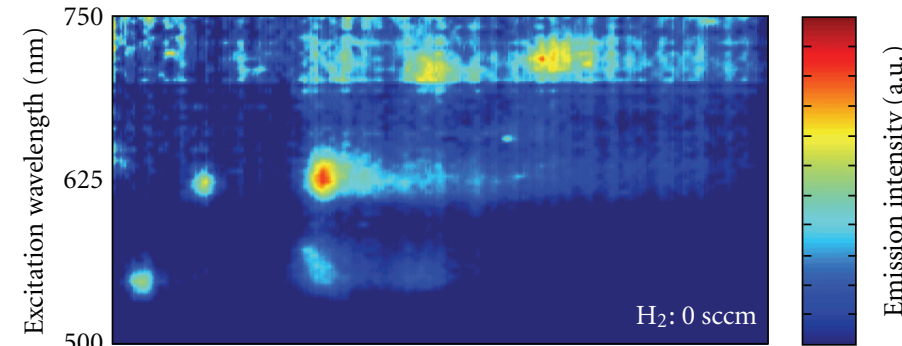

(a)

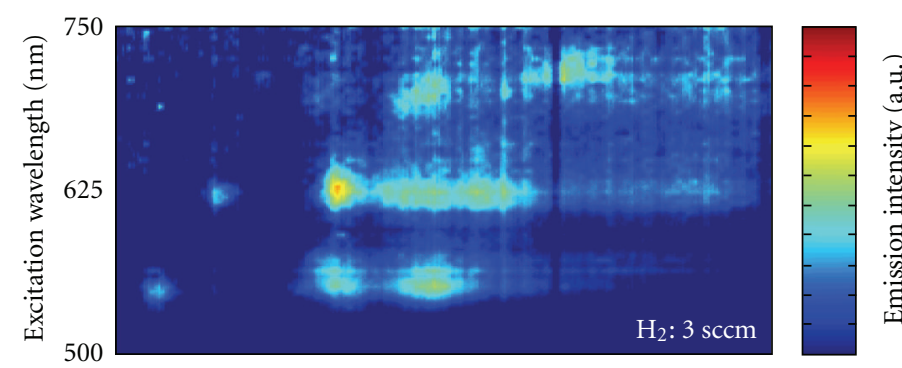

(b)

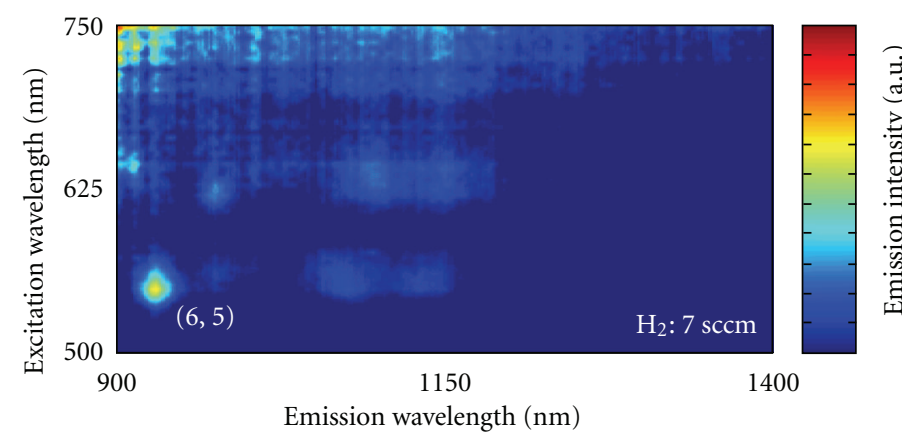

(c)

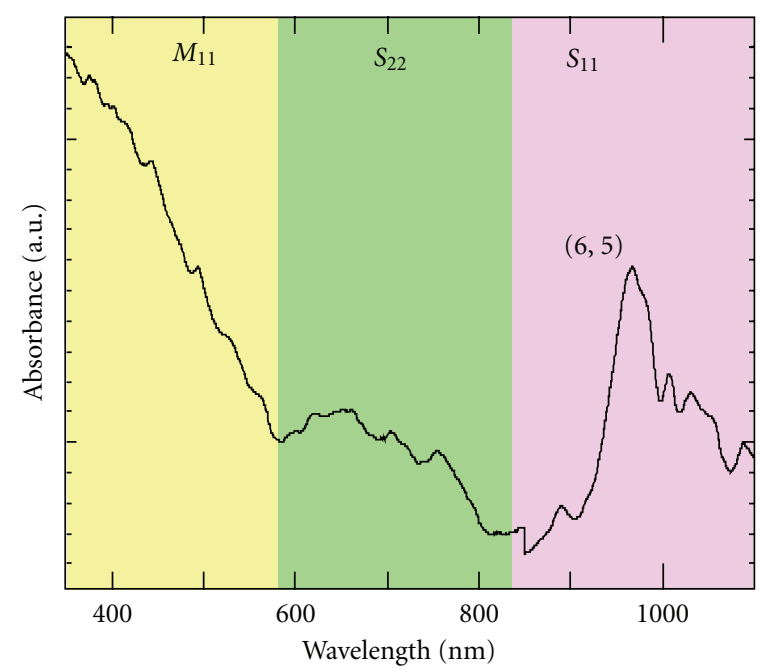

(d)

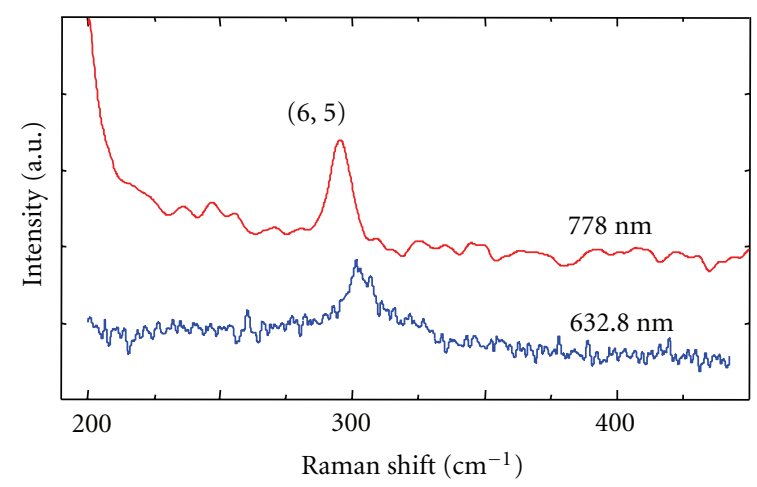

(e)

Figure 4: (a)-(c) PLE maps of SWNTs grown from an Au catalyst by plasma CVD under $\mathrm{H}_{2}$ flow rates of (a) $0 \mathrm{sccm}$, (b) $3 \mathrm{sccm}$, and (c) $7 \mathrm{sccm}$. (d) UV-Vis-NIR spectrum of SWNTs grown from an Au catalyst under a 7-sccm $\mathrm{H}_{2}$ flow rate and (e) Raman spectra of as-synthesized SWNTs grown from an Au catalyst under a $7-\mathrm{sccm} \mathrm{H}_{2}$ flow rate using excitation laser wavelengths of $632.8 \mathrm{~nm}$ and $778 \mathrm{~nm}$.

CVD. This indicates that $\mathrm{H}_{2}$-assisted Au catalyzation is a critical factor for achieving narrow chirality distributions, which is in good agreement with theoretical predictions. The first-principle calculation by Yazyev and Pasquarello reveals that coinage metals, such as $\mathrm{Cu}, \mathrm{Ag}$, and $\mathrm{Au}$, produce narrow chirality distributions [44]. Ding et al. have reported that the SWNT diameter is larger on the surfaces of Fe, $\mathrm{Co}$, and $\mathrm{Ni}$ particles than on $\mathrm{Cu}, \mathrm{Pd}$, and $\mathrm{Au}$ particles because of the different bond energies on the catalyst surfaces [45]. Based on these theoretical models, we can explain the effect of $\mathrm{H}_{2}$-assisted $\mathrm{Au}$ catalyzation on the narrow chirality distribution as follows. Since the binding energy of hydrocarbons on the Au surface is much weaker than on the Fe surface, it is difficult to achieve cap formation for largediameter Au catalysts [45]. Additional $\mathrm{H}_{2}$ also enhances the etching of the carbon precursor from the catalyst surface, which strongly suppresses the growth of large-diameter SWNTs; hence, the chirality distribution of SWNTs grown from the Au catalyst should be narrower than those grown from the $\mathrm{Fe}$ catalyst. The stability of the cap structure is a possible reason why the $(6,5)$ tube was dominant in the small-diameter Au-plasma CVD SWNTs. The number of cap structures, which satisfies the isolated pentagon rule, is highly limited for small-diameter SWNTs, and $(6,5)$ is known to have a stable cap structure in this diameter range [33]. A comparison between Au-plasma CVD and Au-thermal CVD was also carried out. Although the chirality distribution became relatively narrow for SWNTs grown by Au-thermal CVD under appropriate $\mathrm{H}_{2}$ concentrations, it was much broader than that of SWNTs grown by Au-plasma CVD. Comparison of the Au-plasma CVD and Au-thermal CVD processes showed that there were two significant differences in the SWNT growth conditions: growth temperature and incubation time. The lower limit of growth temperature for Au-plasma CVD was $700^{\circ} \mathrm{C}$, which was lower than that of Au-thermal CVD by $50^{\circ} \mathrm{C}$. The initial SWNT growth occurred $1 \mathrm{~min}$ after the growth substrate was exposed to the plasma for Au-plasma CVD, whereas 15 min were required for the growth of SWNTs with Au-thermal CVD. These results suggest that the low-temperature and short-time 
growth with $\mathrm{Au}$-plasma CVD prevents aggregation of catalyst particles during SWNT growth, which suppresses the growth of large-diameter SWNTs and results in a narrow chirality distribution.

5.3. Length Control. Length is another important factor that determines the SWNT properties. Recently, lengthcontrolled SWNTs, especially short SWNTs, have attracted attention because of their unique properties, such as ballistic electron transport [46] and blue-shifted optical band gap due to quantum confinement along the axial direction [47]. These properties have led to applications in high-frequency mechanical resonators [48], molecular sensing [49], biological imaging [50], and drug delivery [50]. However, compared to studies on radial structure control, including tube diameter and chirality, very few efforts have been devoted to axial structure control, that is, length control of SWNTs, especially short SWNTs. Furthermore, simultaneous control of both the radial (diameter and chirality) and axial (length) structure remains a major challenge in fundamental studies and applications of SWNTs.

There are two main approaches used to obtain short SWNTs. One is direct growth, and the other is based on posttreatments, such as cutting [51] and chemical separation [52]. Almost all work reported thus far has used the later approach, which is based on the top-down process. Ultrashort $(<10 \mathrm{~nm})$ SWNTs [47] are fabricated by the combination of chemical cutting and separation. However, SWNTs obtained by these top-down approaches are contaminated by abrasive materials used in some processes, and the use of chemicals and surfactants inhibits the intrinsic property characterization of SWNTs due to reactions and interactions. On the other hand, directlygrown short SWNTs can maintain their original high quality and clean surface with no chemical impurities, making it possible to fully utilize their capabilities in various applications. Very recently, the direct growth of short SWNTs [53] has been reported. However, control of the diameter and chirality of the directly-grown short SWNTs, which is necessary for the fabrication of high-performance electrical and optical devices, has not yet been achieved.

In this subsection, we report a novel direct-growth approach for short SWNTs based on time-programmed plasma CVD. By precisely adjusting the growth time $\left(t_{g}\right)$, the length distribution is narrowed, and short $(<100 \mathrm{~nm})$ SWNTs are successfully grown with very short growth times $(2-5 \mathrm{sec})$. Furthermore, the direct PL study revealed that short SWNTs have the $(6,5)$-rich or $(7,6)$ - and $(8,4)$ dominant narrow chirality distribution. This is the first report for direct growth of short SWNTs with a narrow chirality distribution [54].

Recent progress in in situ TEM observation during SWNT growth revealed that metal-catalyzed SWNT growth is initiated by the formation of a carbon-cap structure on the surface of a catalytic nanoparticle with a certain incubation time $\left(t_{i}\right)$ [55]. Although the detailed mechanism of this incubation period is still debated, it is thought that there might be a correlation between the $t_{i}$ and SWNT structure, such as diameter and chirality. By assuming that the $t_{i}$ of the small-diameter (or specific-chirality) SWNTs is shorter than that of the large-diameter (or other chiralities) SWNTs, it should be possible to selectively grow SWNTs with narrow diameter (or chirality) distributions by strictly controlling the $t_{g}$ of the initial growth stage. Since the length of SWNTs should be proportional to the growth time at the initial growth stage, very short SWNTs can be obtained by adjusting the growth time. For thermal CVD, SWNT growth gradually starts and stops after the feeding and pumping of the hydrocarbon gas, respectively. It is difficult to use thermal CVD for precise growth-time control because there are uncertainties in the growth time. For plasma CVD, on the other hand, reactive ions and radicals are the main species involved in nanotube growth, and SWNT growth occurs only when plasma is generated. This suggests that the growth time can be controlled by tuning the electric power supply used for the plasma generation, and precise $t_{g}$ control on the order of microseconds is possible in plasma CVD. Based on this strategy, we attempted to grow short SWNTs with narrow diameter and chirality distributions by precisely adjusting the $t_{g}$ with time-programmed plasma CVD. Although plasma CVD is a well-known SWNT growth method with advantages of low-temperature growth [17-19] and freestanding growth [14-16], there have been no reports of highly controlled growth time.

The length distribution of SWNTs was carefully investigated by TEM and atomic force microscopy (AFM). For relatively long $(\sim 60 \mathrm{sec})$ growth times, the length distribution of SWNTs was broad (Figures 5(a) and 5(c)). On the contrary, when the growth time was carefully controlled on the order of a few seconds, almost all the SWNTs were very short $(<100 \mathrm{~nm})$, and the distribution also narrowed, as confirmed by direct TEM observation (Figure 5(b)) and AFM characterization (Figure 5(d)). This indicates that it is possible to directly grow short SWNTs by precisely adjusting the growth time with time-programmed plasma CVD. Interestingly, the PLE map of SWNTs grown for long (Figure 5(e)) and short (Figure 5(f)) times showed that the main chirality species were limited to $(7,6)$ and $(8,4)$ (Figure 5(f)) only for short growth times. This indicates that the chirality distribution at the initial growth stage was very narrow.

5.4. Metallicity Control. Thin-film transistors (TFTs) are promising candidates for SWNT nanoelectronics applications. Although high mobility and flexibility make SWNT films ideal for use in various industrial applications, the low on/off current ratio in SWNT TFTs caused by a mixture of metallic and semiconducting SWNTs strongly restricts the practical use of SWNTs in TFTs applications. Recent progress in chemical separation enables us to fabricate highquality devices with on/off ratios of $\sim 10^{4}$ and gate-effective mobility of $\sim 52 \mathrm{~cm}^{2} / \mathrm{Vs}$ [56]. Impurities and defects are occasionally induced in the chemically treated nanotubes during the separation process, which significantly decreases the device performance. Since the as-grown SWNTs maintain their original high quality with low impurity concentrations, the selective growth of semiconducting SWNTs is 


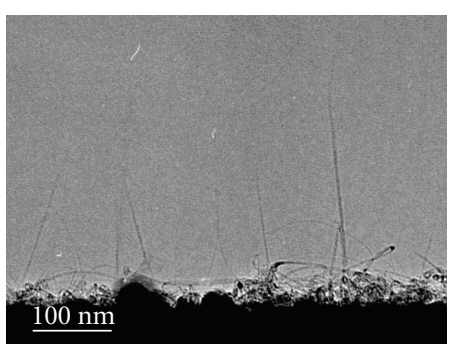

(a)

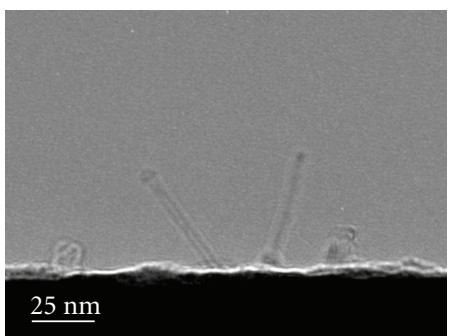

(b)

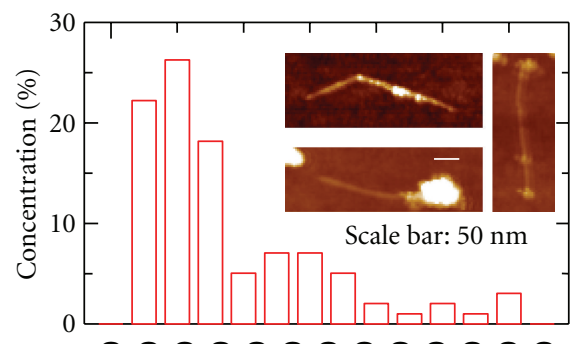

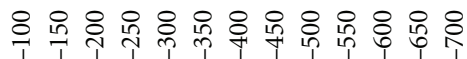

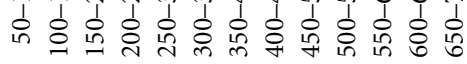

Tube length (nm)

(c)

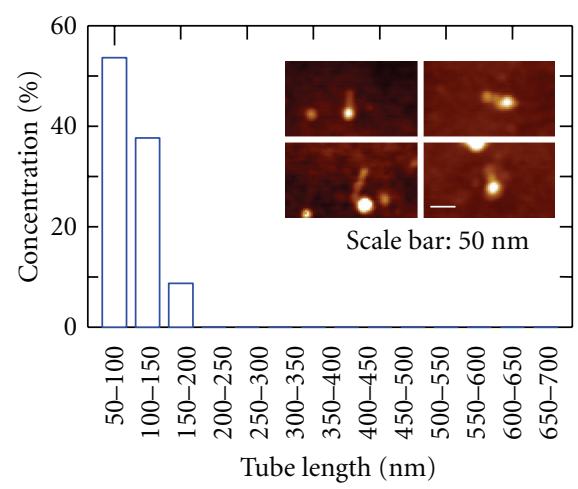

(d)

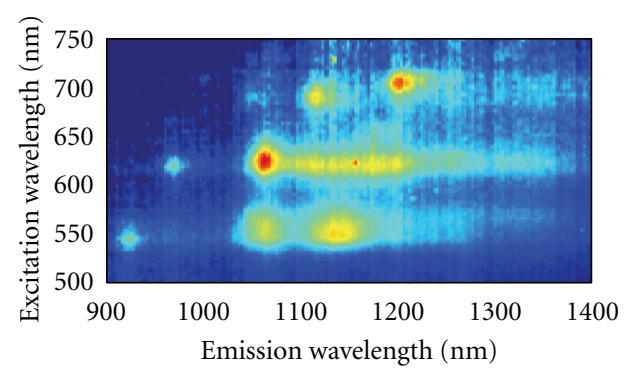

(e)

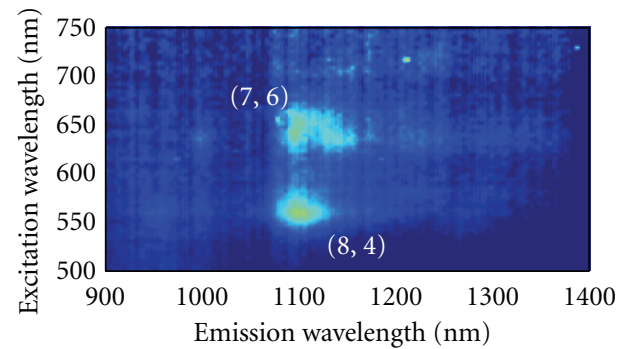

(f)

FIgURE 5: (a, b) TEM images of freestanding SWNTs produced by long (a) and short (b) growth times. (c, d) Length distributions of SWNTs produced by long (c) and short (d) growth times. The inset shows a typical AFM image of SWNTs used for length estimation. (e, f) PLE map of SWNTs grown for long (e) and short (f) times.

an important topic. li et al. reported the preferential growth of semiconducting SWNTs by plasma CVD [57]. Although several similar studies of plasma CVD [58-60] and thermal CVD [61] have also been reported, elucidation of this selective-growth mechanism remains an open question that requires further investigation. Here, we present our recent experimental results on the clear correlation between the performance of semiconducting devices fabricated by plasma CVD-grown SWNTs and the SWNT mean diameter, which might explain the preferential growth of semiconducting SWNTs by plasma CVD.

Figure 6(a) shows typical Raman scattering spectra of SWNTs grown under different growth temperatures. The high G-peak to D-peak ratio indicates that the quality of SWNTs was comparable to that of the conventionally CVD-grown SWNTs. The RBM in the lower wave number region shows the clear down-shift with increasing growth temperature. It is well known that the RBM peak position and tube diameter have a correlation of $\omega=248 / d$, that is, the mean diameter of SWNTs increases with increasing growth temperature. Note that all Raman measurements were carried out for the as-grown SWNTs. The density of SWNTs grown by our system is not so high and they form individual or thin bundled shapes. Thus, the equation for individual SWNTs $(\omega=248 / d)$ is utilized for the diameter estimation. This correlation is thought to be due to the catalyst particle size effect. Higher growth temperatures cause catalyst particle aggregation and increasing catalyst particle size, which result in large-diameter SWNTs. Figures 6(b)-6(d) show a source-drain current $\left(I_{\mathrm{ds}}\right)$ versus gate bias voltage $\left(V_{g}\right)$ curve (Figure $6(\mathrm{~b})$ ), an $I_{\mathrm{ds}}$-source drain voltage $\left(V_{\mathrm{ds}}\right)$ curve (Figure 6(c)), and an SEM image (Figure 6(d)) of TFT devices fabricated with SWNTs grown under $800^{\circ} \mathrm{C}$. The on/off ratio was on the order of $10^{4}$ (Figure 6(b)) with a 3- $\mu \mathrm{A}$ on-current $\left(I_{\mathrm{on}}\right)$ at $V_{\mathrm{ds}}=1 \mathrm{~V}$ (Figure 6(c)). The linear $I_{\mathrm{ds}}$ $V_{\mathrm{ds}}$ characteristic indicates good contact between electrodes and nanotubes. The channel length and width were $2 \mu \mathrm{m}$ and $50 \mu \mathrm{m}$, respectively. A clear dependence was observed from the plot of $I_{\mathrm{on}}$ versus on/off ratio as a function of SWNT growth temperature. The on/off ratio of each device clearly decreased with decreasing growth temperature (Figure 6(e)). The concentration of the working devices, which had on/off ratios greater than 5, was counted and plotted as a function of growth temperature (Figure 6(f)). Interestingly, the working device concentration was only $2.5 \%$ for the $600^{\circ} \mathrm{C}$-grown (small-diameter) SWNTs, whereas more than $90 \%$ of the devices worked for the $800^{\circ} \mathrm{C}$-grown (largediameter) SWNTs. The densities of SWNTs grown under different growth temperatures were similar.

To explain this dependence of the working device concentration on diameter, Ar plasma was irradiated on the devices consisting of small- and large-diameter SWNTs, 


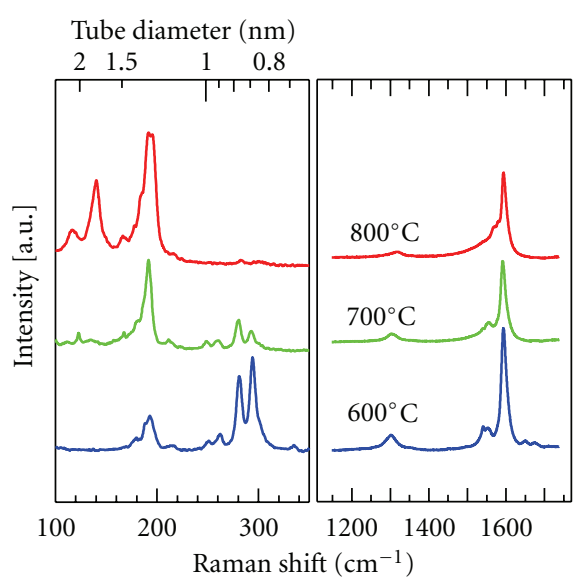

(a)

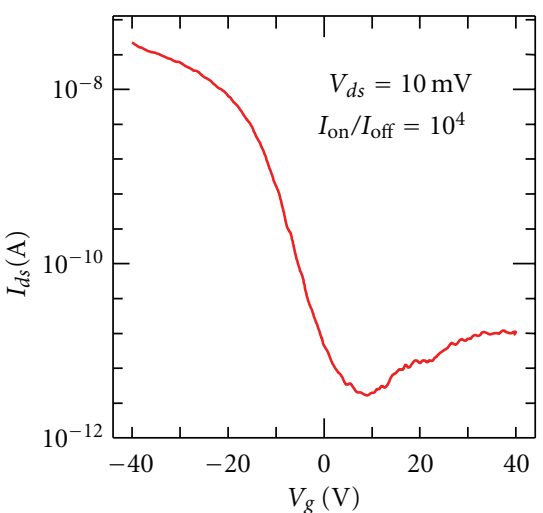

(b)

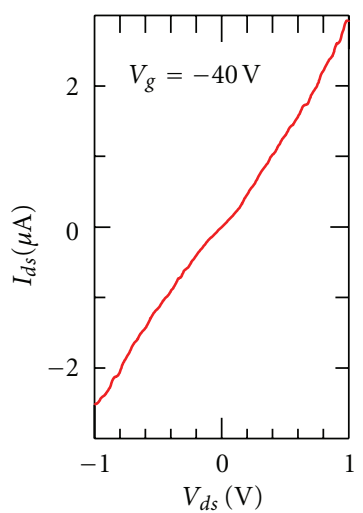

(c)

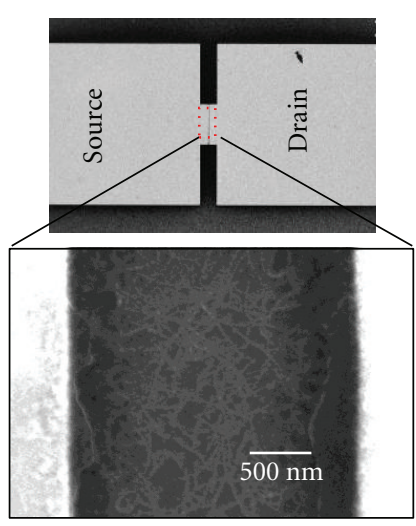

(d)

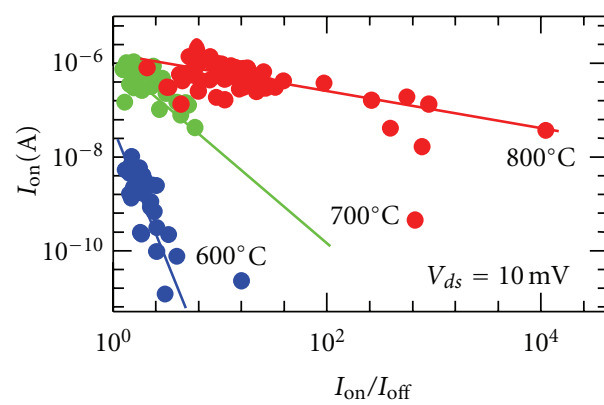

(e)

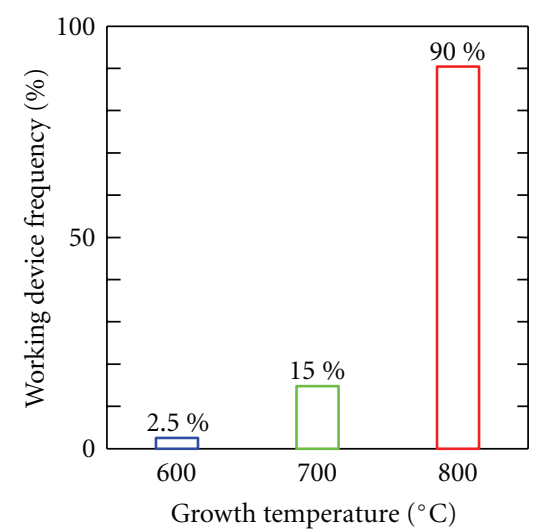

(f)

Figure 6: (a) Raman scattering spectra of SWNTs grown at different growth temperatures. (b) $I_{\mathrm{ds}}-V_{g}$ and (c) $I_{\mathrm{ds}}-V_{\mathrm{ds}}$ characteristics of TFT devices fabricated with SWNTs grown at $800^{\circ} \mathrm{C}$. (d) Typical SEM image of TFT devices with random-network SWNTs grown at $800^{\circ} \mathrm{C}$. (e) $I_{\text {on }}-I_{\text {on }} / I_{\text {off }}$ plot of TFT devices with SWNTs grown at different growth temperatures. (f) Histogram of working device concentration for TFT devices with SWNTs grown at different growth temperatures.

and a defect formation rate was estimated from the current change before and after plasma treatment. For smalldiameter SWNT devices, the on/off ratio did not change, and on- and off-current significantly decreased after Arplasma irradiation. In contrast, the on/off ratio increased with increasing Ar-plasma irradiation time, and the offcurrent depression was significant compared to that of the on-current for large-diameter SWNT devices. Based on these results, the following model can be developed to explain the dependence of the working device concentration on diameter. Due to the curvature effect, small-diameter SWNTs are less stable than large-diameter SWNTs. Hence, both metallic and semiconducting SWNTs are easily deformed by Ar-plasma irradiation with no change in the tube metallicity. On the other hand, in the weak curvature range, metallicity depends on the defect formation rate, which might correlate with reactivity, binding energy between carbon atoms, and a healing process. This model is consistent with the selective etching of metallic SWNTs by a gas-phase reaction, which was previously reported in [62]. Further detailed studies on the selective damage of metallic SWNTs might provide insight on the preferential growth of semiconducting SWNTs by plasma CVD.

\section{Conclusions}

Recent progress in SWNT growth was presented, with a special emphasis on plasma CVD. Due to the strong plasmasheath electric field, it is possible to grow freestanding individual SWNTs by plasma CVD. The growth temperatures for MWNTs and SWNTs were lowered to $\sim 120$ and $400^{\circ} \mathrm{C}$, respectively, by the effective decomposition of carbon source gasses due to energetic electron impact in plasma. Based on the time-evolution study and the detailed plasma parameter measurements, the growth kinetics of SWNTs in plasma CVD were well established. Diameter-, chirality-, and lengthcontrolled growth also also achieved by adjusting the plasma conditions. Moreover, the concentration of semiconducting SWNTs in FET devices can be increased by tuning the mean diameter of SWNTs, and this effect is attributable to selective damage of metallic SWNTs during plasma CVD. 


\section{References}

[1] S. Iijima and T. Ichihashi, "Single-shell carbon nanotubes of 1nm diameter," Nature, vol. 363, no. 6430, pp. 603-605, 1993.

[2] S. J. Tans, M. H. Devoret, H. Dai et al., "Individual singlewall carbon nanotubes as quantum wires," Nature, vol. 386, no. 6624, pp. 474-477, 1997.

[3] S. J. Tans, A. R. M. Verschueren, and C. Dekker, "Roomtemperature transistor based on a single carbon nanotube," Nature, vol. 393, no. 6680, pp. 49-52, 1998.

[4] A. Bachtold, P. Hadley, T. Nakanishi, and C. Dekker, "Logic circuits with carbon nanotube transistors," Science, vol. 294, no. 5545, pp. 1317-1320, 2001.

[5] M. Freitag, J. C. Tsang, J. Kirtley et al., "Electrically excited, localized infrared emission from single carbon nanotubes," Nano Letters, vol. 6, no. 7, pp. 1425-1433, 2006.

[6] T. Guo, P. Nikolaev, A. Thess, D. T. Colbert, and R. E. Smalley, "Catalytic growth of single-walled manotubes by laser vaporization," Chemical Physics Letters, vol. 243, no. 1-2, pp. 49-54, 1995.

[7] J. Kong, A. M. Cassell, and H. Dai, "Chemical vapor deposition of methane for single-walled carbon nanotubes," Chemical Physics Letters, vol. 292, no. 4-6, pp. 567-574, 1998.

[8] S. Maruyama, R. Kojima, Y. Miyauchi, S. Chiashi, and M. Kohno, "Low-temperature synthesis of high-purity singlewalled carbon nanotubes from alcohol," Chemical Physics Letters, vol. 360, no. 3-4, pp. 229-234, 2002.

[9] H. Dai, A. G. Rinzler, P. Nikolaev, A. Thess, D. T. Colbert, and R. E. Smalley, "Single-wall nanotubes produced by metalcatalyzed disproportionation of carbon monoxide," Chemical Physics Letters, vol. 260, no. 3-4, pp. 471-475, 1996.

[10] G. G. Tibbetts, "Growing carbon fibers with a linearly increasing temperature sweep: experiments and modeling," Carbon, vol. 30, no. 3, pp. 399-406, 1992.

[11] Z. F. Ren, Z. P. Huang, J. W. Xu et al., "Synthesis of large arrays of well-aligned carbon nanotubes on glass," Science, vol. 282, no. 5391, pp. 1105-1107, 1998.

[12] M. Chhowalla, K. B. K. Teo, C. Ducati et al., "Growth process conditions of vertically aligned carbon nanotubes using plasma enhanced chemical vapor deposition," Journal of Applied Physics, vol. 90, no. 10, pp. 5308-5317, 2001.

[13] L. Delzeit, C. V. Nguyen, R. M. Stevens, J. Han, and M. Meyyappan, "Growth of carbon nanotubes by thermal and plasma chemical vapour deposition processes and applications in microscopy," Nanotechnology, vol. 13, no. 3, pp. 280-284, 2002.

[14] T. Hirata, N. Satake, G. H. Jeong et al., "Magnetrontype radio-frequency plasma control yielding vertically wellaligned carbon nanotube growth," Applied Physics Letters, vol. 83, no. 6, pp. 1119-1121, 2003.

[15] C. Bower, W. Zhu, S. Jin, and O. Zhou, "Plasma-induced alignment of carbon nanotubes," Applied Physics Letters, vol. 77 , no. 6, pp. 830-832, 2000.

[16] V. I. Merkulov, A. V. Melechko, M. A. Guillorn, D. H. Lowndes, and M. L. Simpson, "Alignment mechanism of carbon nanofibers produced by plasma-enhanced chemicalvapor deposition," Applied Physics Letters, vol. 79, no. 18, pp. 2970-2972, 2001.

[17] S. Hofmann, C. Ducati, J. Robertson, and B. Kleinsorge, "Lowtemperature growth of carbon nanotubes by plasma-enhanced chemical vapor deposition," Applied Physics Letters, vol. 83, no. 1, pp. 135-137, 2003.

[18] YO. S. Min, E. J. Bae, B. S. Oh, D. Kang, and W. Park, "Low-temperature growth of single-walled carbon nanotubes by water plasma chemical vapor deposition," Journal of the American Chemical Society, vol. 127, no. 36, pp. 12498-12499, 2005.

[19] S. Hofmann, C. Ducati, B. Kleinsorge, and J. Robertson, "Direct growth of aligned carbon nanotube field emitter arrays onto plastic substrates," Applied Physics Letters, vol. 83, no. 22, pp. 4661-4663, 2003.

[20] T. Kato, G. H. Jeong, T. Hirata, R. Hatakeyama, K. Tohji, and K. Motomiya, "Single-walled carbon nanotubes produced by plasma-enhanced chemical vapor deposition," Chemical Physics Letters, vol. 381, no. 3-4, pp. 422-426, 2003.

[21] T. Kato, G. H. Jeong, T. Hirata, R. Hatakeyama, and K. Tohji, "Freestanding individual single-walled carbon nanotube synthesis based on plasma sheath effects," Japanese Journal of Applied Physics, vol. 43, no. 10, pp. L1278-L1280, 2004.

[22] T. Kato, R. Hatakeyama, and K. Tohji, "Diffusion plasma chemical vapour deposition yielding freestanding individual single-walled carbon nanotubes on a silicon-based flat substrate," Nanotechnology, vol. 17, no. 9, pp. 2223-2226, 2006.

[23] T. Kato and R. Hatakeyama, "Formation of freestanding single-walled carbon nanotubes by plasma-enhanced CVD," Chemical Vapor Deposition, vol. 12, no. 6, pp. 345-352, 2006.

[24] T. Kato and R. Hatakeyama, "Exciton energy transfer-assisted photoluminescence brightening from freestanding singlewalled carbon nanotube bundles," Journal of the American Chemical Society, vol. 130, no. 25, pp. 8101-8107, 2008.

[25] S. Maruyama, E. Einarsson, Y. Murakami, and T. Edamura, "Growth process of vertically aligned single-walled carbon nanotubes," Chemical Physics Letters, vol. 403, no. 4-6, pp. 320-323, 2005.

[26] M. Suemitsu, Y. Enta, Y. Miyanishi, and N. Miyamoto, "Initial Oxidation of $\mathrm{Si}(100)-(2 \times 1)$ as an Autocatalytic Reaction," Physical Review Letters, vol. 82, no. 11, pp. 2334-2337, 1999.

[27] T. Kato and R. Hatakeyama, "Kinetics of reactive ion etching upon single-walled carbon nanotubes," Applied Physics Letters, vol. 92, no. 3, Article ID 031502, 2008.

[28] T. Kato, S. Kuroda, and R. Hatakeyama, "Diameter tuning of single-walled carbon nanotubes by diffusion plasma CVD," Journal of Nanomaterials, vol. 2011, Article ID 490529, pp. 17, 2011.

[29] A. Jorio, R. Saito, J. H. Hafner et al., "Structural (n, m) determination of isolated single-wall carbon nanotubes by resonant Raman scattering," Physical Review Letters, vol. 86, no. 6, pp. 1118-1121, 2001.

[30] M. S. Dresselhaus, G. Dressehaus, and P. Avouris, Eds., Carbon Nanotubes: Synthesis, Structure, and Applications. Topics in Applied Physics 80, Springer, Berlin, Germany, 2001.

[31] G. Gruner, "Carbon nanonets spark new electronics," Scientific American, vol. 296, no. 5, pp. 76-83, 2007.

[32] B. Kitiyanan, W. E. Alvarez, J. H. Harwell, and D. E. Resasco, "Controlled production of single-wall carbon nanotubes by catalytic decomposition of CO on bimetallic Co-Mo catalysts," Chemical Physics Letters, vol. 317, no. 3-5, pp. 497-503, 2000.

[33] Y. Miyauchi, S. Chiashi, Y. Murakami, Y. Hayashida, and S. Maruyama, "Fluorescence spectroscopy of single-walled carbon nanotubes synthesized from alcohol," Chemical Physics Letters, vol. 387, no. 1-3, pp. 198-203, 2004.

[34] X. Li, X. Tu, S. Zaric et al., "Selective synthesis combined with chemical separation of single-walled carbon nanotubes for chirality selection," Journal of the American Chemical Society, vol. 129, no. 51, pp. 15770-15771, 2007.

[35] W. H. Chiang and R. M. Sankaran, "Linking catalyst composition to chirality distributions of as-grown single-walled 
carbon nanotubes by tuning $\mathrm{Ni}_{x} \mathrm{Fe}_{1-x}$ nanoparticles," Nature Materials, vol. 8, no. 11, pp. 882-886, 2009.

[36] W. Zhou, Z. Han, J. Wang et al., "Copper catalyzing growth of single-walled carbon nanotubes on substrates," Nano Letters, vol. 6, no. 12, pp. 2987-2990, 2006.

[37] D. Takagi, Y. Homma, H. Hibino, S. Suzuki, and Y. Kobayashi, "Single-walled carbon nanotube growth from highly activated metal nanoparticles," Nano Letters, vol. 6, no. 12, pp. 2642 2645, 2006.

[38] D. Takagi, Y. Kobayashi, H. Hibino, S. Suzuki, and Y. Homma, "Mechanism of gold-catalyzed carbon material growth," Nano Letters, vol. 8, no. 3, pp. 832-835, 2008.

[39] S. Bhaviripudi, E. Mile, S. A. Steiner et al., "CVD synthesis of single-walled carbon nanotubes from gold nanoparticle catalysts," Journal of the American Chemical Society, vol. 129, no. 6, pp. 1516-1517, 2007.

[40] Y. Ma, P. O. Lehtinen, A. S. Foster, and R. M. Nieminen, "Magnetic properties of vacancies in graphene and singlewalled carbon nanotubes," New Journal of Physics, vol. 6, pp. $1-15,2004$.

[41] Z. Ghorannevis, T. Kato, T. Kaneko, and R. Hatakeyama, "Growth of single-walled carbon nanotubes from nonmagnetic catalysts by plasma CVD," Japanese Journal of Applied Physics, vol. 49, no. 2, pp. 1-4, 2010.

[42] Z. Ghorannevis, T. Kato, T. Kaneko, and R. Hatakeyama, "Narrow-chirality distributed single-walled carbon nanotube growth from nonmagnetic catalyst," Journal of the American Chemical Society, vol. 132, no. 28, pp. 9570-9572, 2010.

[43] S. M. Bachilo, M. S. Strano, C. Kittrell, R. H. Hauge, R. E. Smalley, and R. B. Weisman, "Structure-assigned optical spectra of single-walled carbon nanotubes," Science, vol. 298, no. 5602, pp. 2361-2366, 2002.

[44] O. V. Yazyev and A. Pasquarello, "Effect of metal elements in catalytic growth of carbon nanotubes," Physical Review Letters, vol. 100, no. 15, Article ID 156102, 2008.

[45] F. Ding, P. Larsson, J. A. Larsson et al., "The importance of strong carbon-metal adhesion for catalytic nucleation of single-walled carbon nanotubes," Nano Letters, vol. 8, no. 2, pp. 463-468, 2008.

[46] A. Javey, J. Guo, Q. Wang, M. Lundstrom, and H. Dai, "Ballistic carbon nanotube field-effect transistors," Nature, vol. 424, no. 6949, pp. 654-657, 2003.

[47] X. Sun, S. Zaric, D. Daranciang et al., "Optical properties of ultrashort semiconducting single-walled carbon nanotube capsules down to sub-10 nm," Journal of the American Chemical Society, vol. 130, no. 20, pp. 6551-6555, 2008.

[48] D. Garcia-Sanchez, A. San Paulo, M. J. Esplandiu et al., "Mechanical detection of carbon nanotube resonator vibrations," Physical Review Letters, vol. 99, no. 8, Article ID 085501, 2007.

[49] K. J. Ziegler, Z. Gu, J. Shaver et al., "Cutting single-walled carbon nanotubes,” Nanotechnology, vol. 16, no. 7, pp. S539S544, 2005.

[50] Z. Liu, S. Tabakman, K. Welsher, and H. Dai, "Carbon nanotubes in biology and medicine: In vitro and in vivo detection, imaging and drug delivery," Nano Research, vol. 2, no. 2, pp. 85-120, 2009.

[51] Z. Chen, K. Kobashi, U. Rauwald et al., "Soluble ultra-short single-walled carbon nanotubes," Journal of the American Chemical Society, vol. 128, no. 32, pp. 10568-10571, 2006.

[52] J. A. Fagan, M. L. Becher, J. Chun, and E. K. Hobbie, "Length fractionation of carbon nanotubes using centrifugation," Advanced Materials, vol. 20, no. 9, pp. 1609-1613, 2008.
[53] B. Liu, W. Ren, C. Liu et al., "Growth velocity and direct length-sorted growth of short single-walled carbon nanotubes by a metal-catalyst- free chemical vapor deposition process," ACS Nano, vol. 3, no. 11, pp. 3421-3430, 2009.

[54] T. Kato and R. Hatakeyama, "Direct growth of short singlewalled carbon nanotubes with narrow-chirality distribution by time-programmed plasma CVD," ACS Nano. In press.

[55] S. Hofmann, R. Sharma, C. Ducati et al., "In situ observations of catalyst dynamics during surface-bound carbon nanotube nucleation," Nano Letters, vol. 7, no. 3, pp. 602-608, 2007.

[56] C. Wang, J. Zhang, K. Ryu, A. Badmaev, L. G. De Arco, and C. Zhou, "Wafer-scale fabrication of separated carbon nanotube thin-film transistors for display applications," Nano Letters, vol. 9, no. 12, pp. 4285-4291, 2009.

[57] Y. Li, D. Mann, M. Rolandi et al., "Preferential growth of semiconducting single-walled carbon nanotubes by a plasma enhanced CVD method," Nano Letters, vol. 4, no. 2, pp. 317321, 2004.

[58] L. Qu, F. Du, and L. Dai, "Preferential syntheses of semiconducting vertically aligned single-walled carbon nanotubes for direct use in FETs," Nano Letters, vol. 8, no. 9, pp. 2682-2687, 2008.

[59] U. J. Kim, E. H. Lee, J. M. Kim, Y. S. Min, E. Kim, and W. Park, "Thin film transistors using preferentially grown semiconducting single-walled carbon nanotube networks by water-assisted plasma-enhanced chemical vapor deposition," Nanotechnology, vol. 20, no. 29, Article ID 295201, 2009.

[60] T. Mizutani, H. Ohnaka, Y. Okigawa, S. Kishimoto, and Y. Ohno, "A study of preferential growth of carbon nanotubes with semiconducting behavior grown by plasma-enhanced chemical vapor deposition," Journal of Applied Physics, vol. 106, no. 7, Article ID 073705, 2009.

[61] L. Ding, A. Tselev, J. Wang et al., "Selective growth of wellaligned semiconducting single-walled carbon nanotubes," Nano Letters, vol. 9, no. 2, pp. 800-805, 2009.

[62] G. Zhang, P. Qi, X. Wang et al., "Selective etching of metallic carbon nanotubes by gas-phase reaction," Science, vol. 314, no. 5801, pp. 974-977, 2006. 

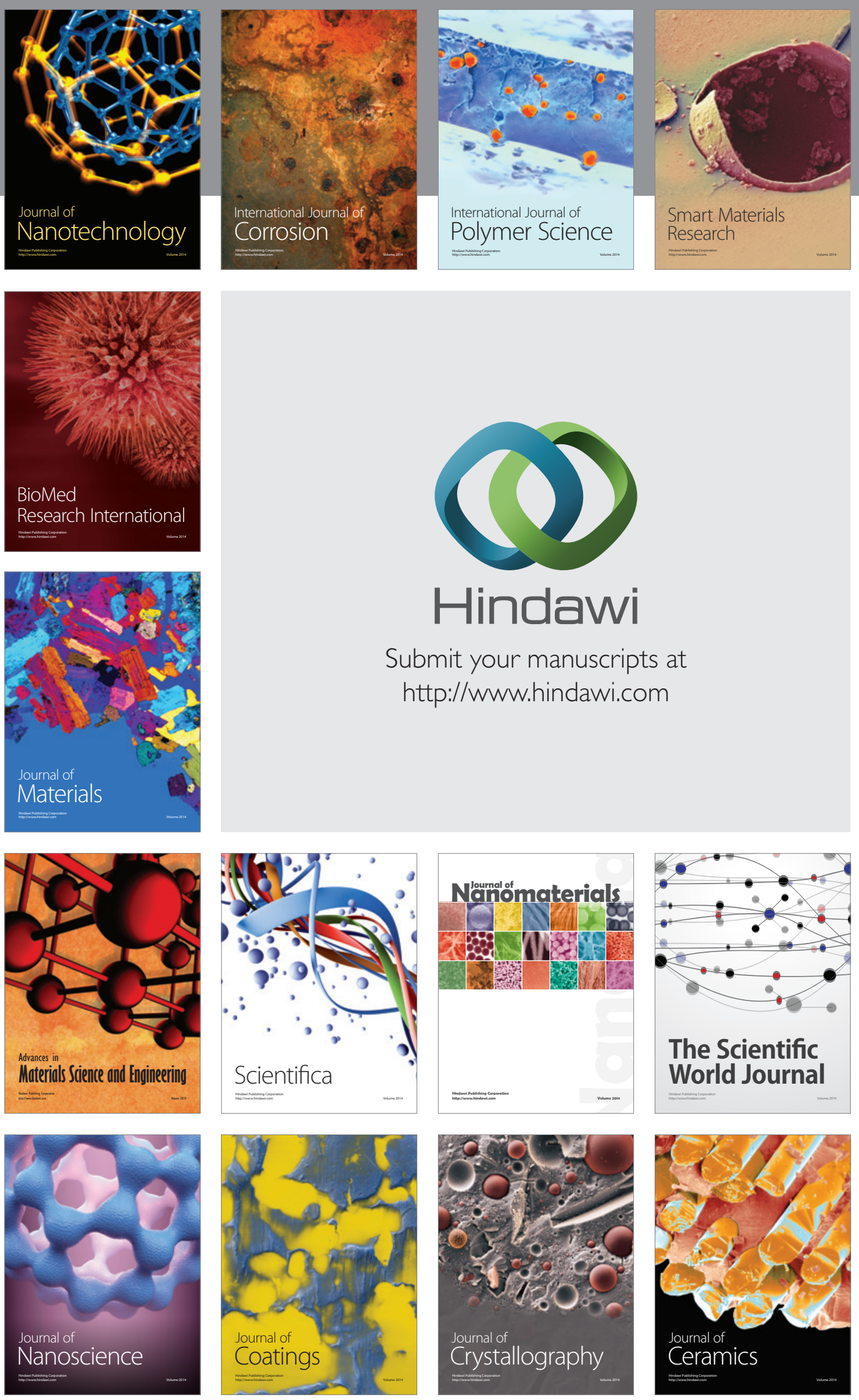

The Scientific World Journal

Submit your manuscripts at

http://www.hindawi.com

\section{World Journal}

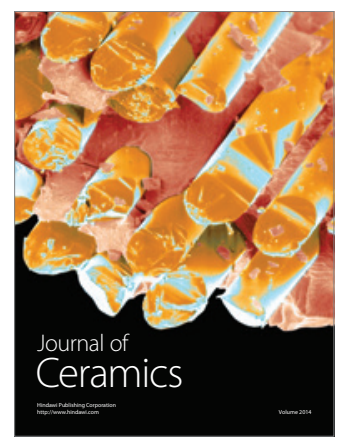

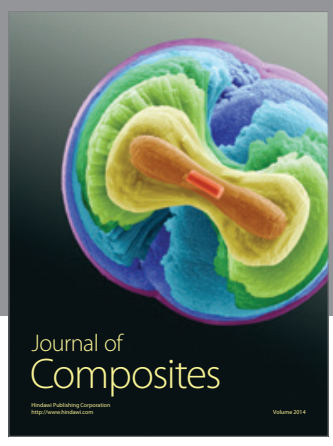
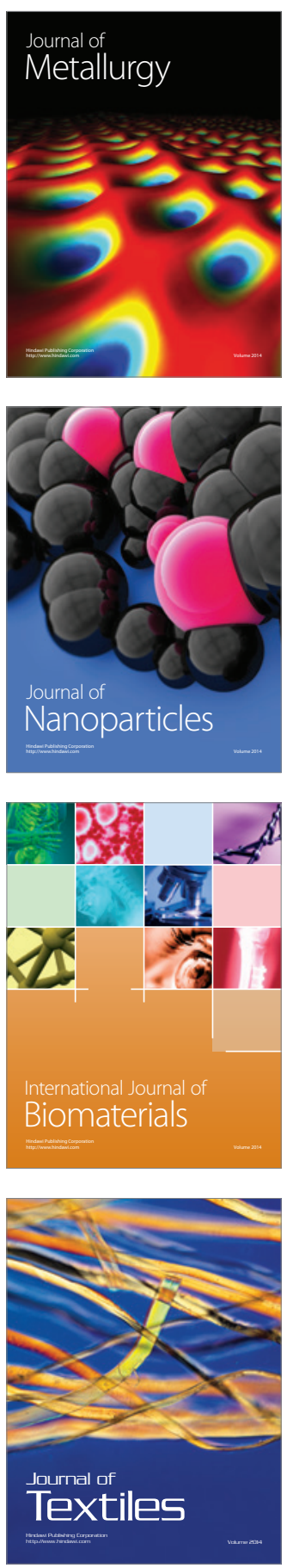